\title{
The Equation of Motion of an Electron: A Debate in Classical and Quantum Physics*
}

\author{
Kwang-Je Kim
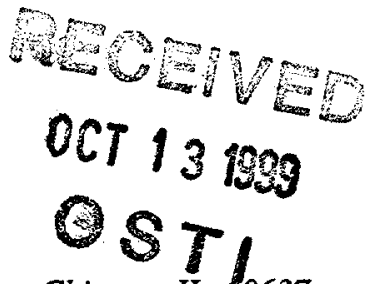 \\ Argonne National Laboratory, Argonne, IL 60439 and The University of Chicago, Chicago, IL 60637
}

\begin{abstract}
The current status of understanding of the equation of motion of an electron is summarized. Classically, a consistent, linearized theory exists for an electron of finite extent, as long as the size of the electron is larger than the classical electron radius. Nonrelativistic quantum mechanics seems to offer a fine theory even in the point-particle limit.
\end{abstract}

\section{INTRODUCTION}

For almost 100 years there has been consideration of the proper equation of motion of an electron. Many fine physicists, beginning with Abraham and Lorentz, have worked on this subject, and there are hundreds of papers in the literature. Here we summarize the present state of understanding, with some historical background, giving the major contributions through the years. This paper is a shortened version of a recent review article written by the author and A. Sessler [1].

In contrast with what most physicists believe, it is seen that the linearized classical theory, when it is applied in the appropriate regime (nonquantum), is in fine shape: it is finite, has no contradiction with relativity, has no runaway solutions, and has no a-causal behavior.

The Abraham-Lorentz equation [2,3] for a point electron involving a third derivative in time suffers from two major problems: contradiction with relativity and runaway and acausal behavior. The work of Poincaré [4] and Dirac [5] solves the problem with relativity. The runaway problem is solved by going to an extended model of an electron described by a difference-differential equation. The equation for the nonrelativistic case was derived by Sommerfeld [6] and Page [7] and was generalized to the relativistic case by Caldirola [8]. The extended model is finite and causal if the electron size $a$ is larger than the classical electron radius $r_{e}=$ $\mathrm{e}^{2} / \mathrm{mc}^{2}=2.7 \times 10^{-13} \mathrm{~cm}$.

\footnotetext{
* Work supported by the U.S. Department of Energy, Office of Basic Energy Sciences, under Contract No. W-31-109-ENG-38.
} 


\section{DISCLAIMER}

This report was prepared as an account of work sponsored by an agency of the United States Government. Neither the United States Government nor any agency thereof, nor any of their employees, make any warranty, express or implied, or assumes any legal liability or responsibility for the accuracy, completeness, or usefulness of any information, apparatus, product, or process disclosed, or represents that its use would not infringe privately owned rights. Reference herein to any specific commercial product, process, or service by trade name, trademark, manufacturer, or otherwise does not necessarily constitute or imply its endorsement, recommendation, or favoring by the United States Government or any agency thereof. The views and opinions of authors expressed herein do not necessarily state or reflect those of the United States Government or any agency thereof. 


\section{DISCLAIMER}

Portions of this document may be illegible in electronic image products. Images are produced from the best available original document. 
Classical theory is clearly not appropriate for examining behavior at a distance less than the Compton wavelength of an electron, $\lambda=\hbar / \mathrm{mc}=4 \times 10^{-11} \mathrm{~cm}$. The work by Moniz and Sharp [9] indicates that in nonrelativistic quantum mechanics an electron behaves as an extended particle with the size of the Compton wavelength: The equation of motion is finite and causal even in the point particle limit as long as the Compton wavelength is larger than the classical electron radius. Furthermore, the mass correction $\delta \mathrm{m}$ is not only finite but actually vanishes in quantum theory. We present a new quantum mechanical derivation of this interesting result [10]. In quantum electrodynamic (QED) analysis, recent work by Low [11] shows that the electron motion is finite in perturbation theory in $\alpha=\mathrm{e}^{2} / \hbar \mathrm{c}$. However, a proper QED analysis has not yet been obtained (and maybe, those do not even exist).

There are several excellent text books $[12,13,14]$ and review articles $[15,16,17]$ on the classical electron theory.

\section{ABRAHAM-LORENTZ AND OTHER CLASSICAL EQUATIONS OF MOTION}

We start by writing various forms of equation of motion of an electron in nonrelativistic classical mechanics. Let the electron trajectory be $\mathbf{y}(\mathrm{t})$. The electron is really a charge distribution centered at $\mathbf{y}(\mathrm{t})$ represented by

$$
\text { ef }(x-y(t))
$$

where $e$ is the electron charge, and $\mathbf{f}(\mathbf{x})$ is a spherically symmetric function, normalized so that

$$
\int f(x) d^{3} x=1
$$

The nonrelativistic equation of motion is determined by the Lorentz force law:

$$
\mathrm{m}_{0} \ddot{\mathbf{y}}(\mathrm{t})=\mathbf{F}_{\mathrm{ext}}+\mathrm{e} \int \mathrm{f}(\mathbf{x}-\mathbf{y}(\mathrm{t}))\left(\mathbf{E}(\mathbf{x})+\frac{1}{\mathrm{c}} \dot{\mathbf{y}}(\mathrm{t}) \times \mathbf{B}(\mathbf{x})\right) \mathrm{d}^{3} \mathrm{x}
$$

where $m_{0}$ is the bare mass, the dot represents the time derivative, $\mathbf{F}_{\text {ext }}$ is the external force, and $\mathbf{E}$ and $\mathbf{B}$ are, respectively, the electric and magnet field in Gaussian units. The second term in the above equation is the electromagnetic self force.

Solving Maxwell's equation to determine the electromagnetic field [11,1], the self force arising from the electric field is found to be

$$
\mathrm{m}_{0} \ddot{\mathbf{y}}(\mathrm{t})=\mathbf{F}_{\text {ext }}(\mathrm{t})-\frac{2}{3} \mathrm{e}^{2} \frac{4 \pi}{(2 \pi)^{3}} \int \mathrm{d}^{3} \mathrm{k}|\tilde{\mathrm{f}}(\mathbf{k})|^{2} \int_{0}^{\infty} \mathrm{d} \tau \mathbf{Y}(\mathrm{t}, \tau) \cos k c \tau,
$$


where

$$
\tilde{f}(k)=\int d^{3} k e^{-i k \cdot x} f(x)
$$

and

$$
Y(t, \tau)=e^{i k \cdot y(t)} e^{i k \cdot y(t-\tau)} \dot{y}(t-\tau)
$$

The force arising from the magnetic field is nonlinear in $\mathbf{y}$ (and its derivative), which we neglect. In linear approximation, the exponential factors in Eq. (5) are replaced by 1. Therefore,

$$
\mathbf{Y}(\mathrm{t}, \tau)=\dot{\mathbf{y}}(\mathrm{t}-\tau)
$$

Equations (4) and (6) are the desired expressions for the classical equation of motion and can be shown to be identical to the power series expression derived by Lorentz for linearized radiation reaction in the nonrelativistic approximation $[3,12]$.

For the case of a spherical shell of radius a,

$$
f(x)=\frac{\delta(|x|-a)}{4 \pi a^{2}}, \tilde{f}(k)=\frac{\sin k a}{k a} .
$$

Equations (4) and (6) become

$$
\mathrm{m}_{0} \ddot{\mathbf{y}}(\mathrm{t})=\mathrm{F}_{\mathrm{ext}}(\mathrm{t})+\frac{\mathrm{e}^{2}}{3 \mathrm{a}^{2} \mathrm{c}}[\dot{\mathbf{y}}(\mathrm{t}-2 \mathrm{a} / \mathrm{c})-\dot{\mathbf{y}}(\mathrm{t})]
$$

This differential-difference equation will be referred to as the Sommerfeld-Page equation because they derived it $[6,7]$.

Expanding $\dot{\mathbf{y}}(\mathrm{t}-2 \mathrm{a} / \mathrm{c})$ in Eq. (8) in a Taylor series and neglecting terms that vanish as $\mathrm{a} \rightarrow 0$,

$$
\mathrm{m}_{0} \ddot{\mathbf{y}}(\mathrm{t})=\mathbf{F}_{\text {ext }}(\mathrm{t})-\delta \mathrm{m} \ddot{\mathbf{y}}(\mathrm{t})+\frac{2 \mathrm{e}^{2}}{3 \mathrm{c}^{3}} \dddot{\mathrm{y}}(\mathrm{t})
$$

where

$$
\delta \mathrm{m}=\frac{4}{3} \frac{\mathrm{e}^{2}}{2 \mathrm{ac}^{2}}
$$

Equation (9) will be referred to as the Abraham-Lorentz equation in the following. It is the simplest form of the equation of motion, taking into account the electromagnetic self force in a nonrelativistic linear approximation and in the point-particle limit. 


\section{PROBLEM WITH RELATIVITY AND POINCARÉ STRESS}

The second term on the RHS of the Abraham-Lorentz equation can be interpreted as the inertia due to the electromagnetic mass $\delta \mathrm{m}$. However, it is in contradiction with the notion of relativity because $\delta \mathrm{m}$ is different from the electrostatic mass $\mathrm{e}^{2} / 2 \mathrm{ac}^{2}$ by a factor of $4 / 3$.

To see the meaning of the third derivative term in the Abraham-Lorentz equation, we move the $\delta \mathrm{m}$ term to the LHS, multiply both sides by $\dot{\mathbf{y}}$, and integrate over a finite interval of time. The LHS becomes the increase in the electron's kinetic energy. The RHS is

$$
-\frac{2 \mathrm{e}^{2}}{3 \mathrm{c}^{3}} \int_{\mathrm{t}_{1}}^{t_{2}} \ddot{\mathbf{y}}^{2} \mathrm{dt}+\left.\frac{2 \mathrm{e}^{2}}{3 \mathrm{c}^{3}} \dot{\mathbf{y}} \cdot \ddot{\mathbf{y}}\right|_{\mathrm{t}_{1}} ^{t_{2}}
$$

As long as the second term is negligible, this is the negative of the well-known dipole radiation. The last term in Eq. (9) is therefore reasonable. However, we will see later that this term gives rise to runaway or acausal behavior.

Equation (9) therefore contains two major difficulties: i) a contradiction with relativity, and ii) runaway and preacceleration behavior. We shall see in the following how these troubles are avoided. The bottom line is that there is no problem if the shell radius $a$ is larger than the classical electron radius $r_{e}$.

In 1903, before special relativity was established, Abraham derived [2] the force equation, which is the relativistic generalization of Eq. (9). Abraham also found the rate of work done on an electron, the power equation. Although these equations were derived before relativity was fully established, he used the correct relativistic model of an electron in which the spherical charge distribution in the rest frame is contracted to a spheroid for a moving electron. The derivations of these equations are difficult and confirmed by Schott [18] who carried out a very rigorous and complicated calculation.

It is not necessary to write down these equations here. However, we remark that Abraham's power equation for nonrelativistic speed becomes

$$
\frac{\mathrm{dE}}{\mathrm{dt}}=\mathbf{F}_{\mathrm{ext}} \cdot \dot{\mathbf{y}}-\frac{5}{6} \frac{\mathrm{e}^{2}}{\mathrm{ac}^{2}} \dot{\mathbf{y}} \cdot \ddot{\mathbf{y}}+\frac{2}{3} \frac{\mathrm{e}^{2}}{\mathrm{c}^{3}} \dot{\mathbf{y}} \cdot \dddot{\mathbf{y}} .
$$

Note that $\dot{\mathbf{y}}$ - force (Eq. (9)) does not yield the power (Eq. (12)). This is a fundamental difficulty. The problem was solved by Poincaré [4] with a nonelectromagnetic stress and Dirac by invoking covariance [5].

Poincaré, in his paper submitted in 1905 [4] (without knowledge of Einstein's work on special relativity), observed that a purely electromagnetic model of the electron, such as the charged sphere, is not internally consistent because it will fly apart due to the electrostatic repulsion. To counteract the repulsive force, he imagined that the inside of the sphere provides a uniform negative pressure (or stress ) $p=e^{2} / 8 \pi a^{4}$. 
Relative contributions of Poincaré and Einstein to special relativity is a subject of some debate and considerable historical interest. See A.A. Longunov referred to in [4].

To see how this Poincare stress solves the problem, consider the charged shell in motion. Due to the Lorentz contraction, the sphere becomes an oblate spheroid with the minor axis in the direction of the motion reduced by $\gamma=1 / \sqrt{1-\dot{y}^{2} / c^{2}}$. The work done by the mechanical force is given by the pressure times the volume change. (The pressure is relativistically invariant since the force and the area element transform the same way.) Thus the mechanical system must loose energy at the rate

$$
\begin{aligned}
\frac{\mathrm{dE}}{\mathrm{dt}} & =-\frac{\mathrm{e}^{2}}{8 \pi \mathrm{a}^{4}} \frac{\mathrm{d}}{\mathrm{dt}}\left(\frac{1}{\gamma} \frac{4}{3} \pi \mathrm{a}^{3}\right) \\
& =-\frac{2}{3} \frac{\mathrm{e}^{2}}{\mathrm{a}} \frac{\mathrm{d}}{\mathrm{dt}} \frac{1}{4 \gamma} .
\end{aligned}
$$

Here the expression $4 \pi \mathrm{a}^{3} / 3 \gamma$ is the volume of the spheroid. In the nonrelativistic approximation Eq. (13) becomes $\frac{\mathrm{dE}}{\mathrm{dt}}=\left(\frac{1}{6}\right)\left(\frac{\mathrm{e}^{2}}{\mathrm{a}}\right) \dot{\mathrm{y}} \bullet \ddot{\mathrm{y}} / \mathrm{c}^{2}$. If this is added to Eq. (12), the discrepancy between the force and the power equation is removed. It can be shown that the relativistic loss of mechanical energy, Eq. (13), removes a similar difficulty in relativistic force and power equations [15,1].

However, the problem of the mass-the fact that the electromagnetic mass is $4 / 3$ times the electrostatic mass-is not solved yet. One way of solving the problem is to say that the bare mass contains a term -(1/3) times electrostatic mass. For a more formal approach [19], we can introduce a stress tensor representing the Poincaré stress by

$$
\theta_{\text {Poincare }}^{\mu v}=\frac{e^{2}}{8 \pi a^{3}}\left(g^{\mu v}-q u^{\mu} u^{v}\right) \Theta(a-r)
$$

where $\mathrm{g}^{\mu \mathrm{v}}=(1,-1,-1,-1)$, $\mathrm{q}$ is an arbitrary constant, $\mathrm{u}^{\mu}$ is the velocity four-vector, $\Theta$ is the step function, and $\mathrm{r}$ is the radial coordinate in the rest frame of the shell. The quantity $\theta_{\text {Poincare }}^{\mu v}$ is constructed so that

$$
\partial_{\mu} \theta^{\mu v}=\partial_{\mu}\left(\theta_{\mathrm{EM}}^{\mu v}+\theta_{\text {Poincare }}^{\mu v}\right)=0
$$

where $\theta_{\mathrm{EM}}^{\mu v}$ is the electromagnetic stress tensor associated with the Coulomb field of a spherical shell. Equation (15) assures that the total momentum 


$$
p^{\mu}=\int d^{3} x \theta^{\mu o}
$$

is a four vector [12]. The momentum associated with the Poincare stress alone is

$$
\mathrm{p}_{\text {Poincare }}^{\mu}=\int \mathrm{a}^{3} \mathrm{x} \theta_{\text {Poincare }}^{\mu \mathrm{o}}=+\frac{\mathrm{e}^{2}}{2 \mathrm{a}} \frac{1}{3}\left[-\frac{1}{\gamma}+\mathrm{q} \gamma, \mathrm{q} \gamma \mathbf{u}\right] .
$$

We need to add $\mathrm{dp}_{\text {Poincare }}^{\mu} / \mathrm{dt}$ to the force and the power equations. Our previous result (Eq. (13)) corresponds to the case $q=0$. If, on the other hand, we choose $q=1$, we remove the so-called $4 / 3$ problem.

The above method of solving the $4 / 3$ problem is clearly rather formal and arbitrary. There are also more intuitive approaches, for example by Boyer [20] who notes that the Poincare stress may not act at the same time in all parts of the moving electron. A review of different solutions of the $4 / 3$ problem is given by Rohrlich [17].

The saga of the $4 / 3$ problem is in some sense a story of how special relativity proved itself as a theory of internal consistency and beauty. The investigation of the electron's equation of motion started while special relativity was still evolving. Therefore, there was doubt whether the electron theory was consistent with relativity, which lingered even after relativity was fully established. Einstein, never doubting relativity, wasted no time in checking covariance of the electron theory. He was too busy working out general relativity!

With this choice of $q$, the force-power equation in the relativistic case becomes

$$
m_{0} c^{2} \frac{d}{d s} u^{u}=f_{e x t}^{\mu}+\Gamma^{\mu}-\frac{e^{2}}{2 a} \frac{d u^{\mu}}{d s}
$$

where

$$
\Gamma^{\mu}=\frac{2}{3} \mathrm{e}^{2}\left(\frac{\mathrm{d}^{2} \mathrm{u}^{\mu}}{\mathrm{ds}^{2}}+\left(\frac{\mathrm{du}}{\mathrm{ds}}^{\alpha} \frac{\mathrm{du} \alpha}{\mathrm{ds}}\right) \mathrm{u}^{\mu}\right) .
$$

We now have a relativistically covariant equation of motion of an electron with the observed mass $\mathrm{m}=\mathrm{m}_{0}+\delta \mathrm{m}$ and $\delta \mathrm{m}=\mathrm{e}^{2} / 2 \mathrm{ac}^{2}$.

Before closing this section, we need to mention Dirac's contribution [5]. He understood that the problem with the Abraham-Lorentz equation was in trying to approach too near an electron. He therefore devised an ingenious way to avoid the difficulty. Stay a finite distance away from an electron and demand relativistic covariance. He then obtained Eq. (18), which is therefore referred to as the AbrahamLorentz-Dirac equation. 
Actually, Dirac's derivation of Eq. (18) is valid only up to a term of the form $\mathrm{dB}^{\mu} / \mathrm{ds}$, where $\mathrm{B}^{\mu}$ is a four vector with the restriction $\mathrm{u}^{\mu} \mathrm{dB}_{\mu} / \mathrm{ds}=0$. The simplest choice, $\mathrm{B}^{\mu}=\mathrm{ku}^{\mu}$, gives rise to a term that can be incorporated into the inertial term (mc $\mathrm{du}^{\mu} / \mathrm{ds}$ ) in Eq. (18). The next order term involving $\mathrm{du}^{\mathrm{H}} / \mathrm{ds}$ is complicated, which is disposed of by saying that a simple thing like an electron cannot possibly have such a complication.

If we are willing to make a few very plausible assumptions, the Abraham-LorentzDirac equation can be derived very easily as follows [17]:

$$
\mathrm{m}_{0} \mathrm{c}^{2} \frac{\mathrm{du}^{\mu}}{\mathrm{ds}}=\mathrm{f}_{\mathrm{ext}}^{\mu}+\mathrm{X}^{\mu},
$$

where $X^{\mu}$ includes radiative effects. Both sides of Eq. (20) are orthogonal to $u^{\mu}$. Thus we may write $X^{\mu}=\left(g^{\mu \nu}-u^{\mu} u^{v}\right) Y_{v}$. Try $Y^{\mu}$ of the form $a u^{\mu}+b d u^{\mu} / d s+c d^{2} u^{\mu} / d s^{2}$. The first term does not contribute, and the second term is of the same form as the mass term, which can be incorporated into $\mathrm{m}$. With the third term, it is easy to show that $\mathrm{X}^{\mu}$ is proportional to $\Gamma^{\mu}$. (Here we are using $(\mathrm{du} / \mathrm{ds}) \mathrm{du}_{\alpha} / \mathrm{ds}+\mathrm{d}^{2} \mathrm{u}_{\alpha} / \mathrm{ds}^{2} \cdot \mathrm{u}^{\alpha}=0$.) The coefficient is determined by demanding consistency with the nonrelativistic AbrahamLorentz equation, leading to $\Gamma^{\mu}=X^{\mu}$. End of the proof.

\section{RUNAWAY AND ACAUSALITY}

The general solution of the Abraham-Lorentz equation is

$$
\mathrm{m} \ddot{\mathbf{y}}(\mathrm{t})=\mathrm{e}^{\mathrm{t} / \mathrm{t}_{\mathrm{e}}}\left[\mathrm{m} \ddot{\mathbf{y}}(0)-1 / \mathrm{t}_{\mathrm{e}} \int_{0}^{\mathrm{t}} \mathrm{dt^{ \prime }} \mathrm{e}^{-t / \mathrm{e}_{\mathrm{e}}} \mathbf{F}_{\mathrm{ext}}\left(\mathrm{t}^{\prime}\right)\right]
$$

where

$$
\mathrm{t}_{\mathrm{e}}=\frac{2}{3} \frac{\mathrm{r}_{\mathrm{e}}}{\mathrm{c}}=\frac{2}{3} \frac{\mathrm{e}^{2}}{\mathrm{mc}^{3}} .
$$

The solution in general exhibits exponential growth, i.e., the runaway behavior. Dirac noted that the runaway can be avoided if we choose the initial condition:

$$
\mathrm{m} \ddot{\mathbf{y}}(0)=1 / \mathrm{t}_{\mathrm{e}} \int_{0}^{\infty} \mathrm{dt} \mathrm{e}^{-\mathrm{t}^{\prime} / \mathrm{t}_{\mathrm{e}}} \mathbf{F}_{\mathrm{ext}}\left(\mathrm{t}^{\prime}\right)
$$


Then

$$
m \ddot{\mathbf{y}}(t)=\int_{0}^{\infty} d \alpha \mathrm{e}^{-\alpha} F_{\text {ext }}\left(t+t_{e} \alpha\right)
$$

This solution now exhibits preacceleration: the particle starts to move before the force is applied and the initial condition depends upon the entire future path.

Nevertheless, it satisfies the Rohrlich criteria of "the unobservability of very small charges" [14]. That is, as the charge becomes very small we shouldn't have a solution widely different from that of an uncharged particle.

The Abraham-Lorentz equation exhibits runaway and preacceleration because, as the electron radius a vanishes, the bare mass $m_{0}$ becomes negative to keep the observed mass $m$ finite. A particle with a negative mass can clearly supply an infinite amount of energy.

The difficulty can be avoided in an extended electron model. To see this, we return to the Sommerfeld-Page Eq. (8), which can be written in the following form $[9,16]$ :

$$
\ddot{\mathbf{y}}(\mathrm{t})=\frac{\mathrm{F}_{\mathrm{ext}}(\mathrm{t})}{\mathrm{m}\left(1-\mathrm{ct}_{\mathrm{e}} / \mathrm{a}\right)}+\left(\frac{\mathrm{c}}{2 \mathrm{a}}\right)\left(\frac{\mathrm{ct} \mathrm{e}_{\mathrm{e}}}{\mathrm{a}}\right) \frac{1}{\left(1-c \mathrm{t}_{\mathrm{e}} / \mathrm{a}\right)}[\dot{\mathbf{y}}(\mathrm{t}-2 \mathrm{a} / \mathrm{c})-\dot{\mathbf{y}}(\mathrm{t})] \text {. }
$$

In the above expression, the combination $\mathrm{m}\left(1-\mathrm{ct}_{\mathrm{d}} / \mathrm{a}\right)$ is simply the bare mass $\mathrm{m}_{0}$. Assume that there are no external forces, and there exists a runaway solution of the form $y(t)=y(0) e^{\alpha t}$ with a positive real part of $\alpha$. From Eq. (25) it is easy to see that this is possible only when $1-\mathrm{ct}_{\mathrm{e}} / \mathrm{a}$ is negative. Thus the runaway solution is possible if and only if the electron radius $a$ is less than $(2 / 3) r_{e}$. For $a>(2 / 3) r_{e}$ we get damped oscillatory solutions.

It is sometimes stated that the runaway behavior is due to the infinite energy associated with a point electron. This is false because the runaway occurs for even a finite a as long as it is less than $(2 / 3) r_{e}$. Runaway occurs if and only if the bare mass is negative (and, therefore, the Hamiltonian is no longer positive definite).

We can also show that if $a>c t_{e}$, the motion is causal with no preacceleration. This can be seen most easily if Eq. (25) is turned into an integral equation with a Green's function $[9,16]$ :

$$
\ddot{\mathbf{y}}(\mathrm{t})=\int_{-\infty}^{\infty} \mathrm{G}\left(\mathrm{t}-\mathrm{t}^{\prime}\right) \mathbf{F}_{\mathrm{ext}}\left(\mathrm{t}^{\prime}\right) d \mathrm{t}^{\prime}
$$

where

$$
G\left(t-t^{\prime}\right)=\frac{1}{2 \pi m} \int_{-\infty}^{\infty} \frac{e^{\left.-i \omega x t-t^{\prime}\right)} d \omega}{B} \text {, and }
$$




$$
B=1-\frac{i}{\omega}\left(\frac{c}{2 a}\right)\left(\frac{c t_{e}}{a}\right)\left[e^{\frac{i \omega 2 a}{c}}-\frac{i \omega 2 a}{c}-1\right] .
$$

It can be shown that all poles of $1 / \mathrm{B}(\omega)$ occur in the lower half $\omega$-plane if

$$
a>\frac{2}{3} r_{e}
$$

Then the Green's function $G(\tau)$ vanishes for $\tau<0$. Therefore, the motion is causal. On the other hand, if $a<\frac{2}{3} r_{e}$, then there is in general acausal behavior.

A relativistic generalization of the Sommerfeld-Page equation was conjectured by Caldirola [8] and proved by Yaghjian [15]:

$$
\mathrm{m}_{0} \mathrm{c}^{2} \dot{\mathrm{u}}^{\mu}=\mathrm{f}_{\mathrm{ext}}^{\mu}+\frac{2}{3} \frac{\mathrm{e}^{2}}{\mathrm{a}} \frac{1}{2 \mathrm{a}}\left[\mathrm{u}^{\mu}(\mathrm{s}-2 \mathrm{a})-\mathrm{u}^{\mu}(\mathrm{s}) \mathrm{u}_{\alpha}(\mathrm{s}) \mathrm{u}^{\alpha}(\mathrm{s}-2 \mathrm{a})\right] \text {. }
$$

The Caldirola equation is fine: no runaways and no causality problems. However, when we take $a \rightarrow 0$, then there are problems.

Yaghjian [15] attempted to produce a causal equation with no runaways for a point electron. He modified the Abraham-Lorentz Eq. (9) by multiplying the third derivative term by a function $\eta(t)$, which changes smoothly from 0 for $t<0$ to 1 for $t>2 \mathrm{a} / \mathrm{c}$ (or by multiplying the $\Gamma^{\mu}$ term by a similar function $\eta(s)$ ), arguing that the Taylor series expansion breaks down when the force changes abruptly. However, the solution of the modified equation still exhibits acausal behavior.

\section{QUANTUM MECHANICAL ANALYSIS}

It had been hoped that the difficulty of the classical theory in taking a point particle limit might be solved by quantum mechanics. The observation that the mass renormalization $\delta \mathrm{m}$ is less singular in quantum electrodynamics than in classical theory seemed to reinforce this hope. However, a real quantum mechanical analysis of the electron equation has not been carried out until modern time. The reason behind this lack of activity appears to be the success of the renormalization theory in quantum electrodynamics, which established that all observable phenomena can be calculated to a finite answer order by order in perturbation theory in terms of the observed mass and charge.

In 1977, Moniz and Sharp presented a very interesting quantum mechanical calculation [9], according to which the equation of motion in nonrelativistic quantum mechanics is finite even in the point particle limit. The derivation is based on the elaborate manipulations of infinite series.

Here another derivation will be summarized [10]. 
The derivation of the quantum mechanical equation of motion for Heisenberg operators proceeds similarly to the derivation in the classical case given in section 2 . Again neglecting the magnetic term, one arrives at an equation identical in form to Eq. (4), but the operator $Y$ is given by the symmetrized version of the classical expression, Eq. (5), as follows:

$$
\begin{gathered}
\mathrm{Y}(\mathrm{t}, \tau)=\frac{1}{4}\left\{\mathrm{e}^{\mathrm{ik} \cdot \mathbf{y}_{2}},\left\{\mathrm{e}^{-\mathrm{ik} \cdot \mathbf{y}_{1}}, \dot{\mathbf{y}}_{1}\right\}_{+}\right\}_{+} \\
=\frac{1}{4}\left(\mathrm{e}^{\mathrm{ik} \cdot \mathbf{y}_{2}} \mathrm{e}^{-\mathrm{ik} \cdot \mathbf{y}_{1}}\right)\left(\dot{\mathbf{y}}_{1}+\mathrm{e}^{\mathrm{ik} \cdot \mathbf{y}_{1}} \dot{\mathbf{y}}_{1} \mathrm{e}^{-\mathbf{i k} \cdot \mathbf{y}_{1}}\right)+[\mathbf{k} \rightarrow-\mathbf{k}]^{+} .
\end{gathered}
$$

Here $\mathbf{y}_{2}=\mathbf{y}(t), \mathbf{y}_{1}=\mathbf{y}(t-\tau),\{\quad\}_{+}$is the anticommutator, and $[\mathbf{k} \rightarrow-\mathbf{k}]^{+}$indicates terms obtained by changing the sign of $\mathbf{k}$ and taking the Hermitian conjugate.

Noting that $\mathbf{P}=\mathbf{P}-\mathrm{eA} / \mathrm{c}=\mathrm{m}_{0} \dot{\mathbf{y}}$ is the kinetic momentum operator $(\mathbf{P}=$ canonical momentum), we obtain

$$
\mathrm{e}^{\mathrm{ik} \cdot \mathbf{y}_{1}} \dot{\mathbf{y}}_{1} \mathrm{e}^{-\mathrm{ik} \cdot \mathrm{y}_{1}}=\dot{\mathbf{y}}_{1}-\mathrm{ck} \lambda,
$$

where $\lambda=\hbar / \mathrm{m}_{0} \mathrm{c}$ is the Compton wavelength for the bare mass $\mathrm{m}_{0}$. In classical mechanics, we would have replaced the factor $\exp \left(\mathbf{i k} \cdot \mathbf{y}_{1}\right.$ ) by 1 since it would at most contribute to nonlinear terms. Such a procedure is not justified in quantum mechanics as is clear from Eq. (32). Similarly, the factor $\exp \left(\mathbf{i k} \cdot \mathbf{y}_{2}\right) \cdot \exp \left(-\mathbf{i k} \cdot \mathbf{y}_{1}\right)$ in Eq. (31) cannot simply be replaced by 1 . Instead, we reduce the product of exponential operators using a technique similar to that used by Baier and Katkov [21] in their calculation of quantum synchrotron radiation. The steps used here follow closely a simplified formulation of the Baier-Katkov reduction by Cahn and Jackson [22]. We write

$$
\begin{aligned}
\exp \left(\mathbf{i k} \cdot \mathbf{y}_{2}\right) \exp \left(-\mathrm{ik} \cdot \mathbf{y}_{1}\right) & =\exp (\mathrm{i} \tau \mathrm{H} / \hbar) \exp \left(\mathrm{ik} \cdot \mathbf{y}_{1}\right) \exp (-\mathrm{i} \tau \mathrm{H} / \hbar) \exp \left(-\mathbf{i k} \cdot \mathbf{y}_{1}\right) \\
& =\exp (\mathrm{i} \tau \mathrm{H}(\mathbf{p}) / \hbar) \exp (-\mathrm{i} \tau \mathrm{H}(\mathbf{p}-\hbar \mathrm{k}) / \hbar) \quad\left(\tau=\mathrm{t}-\mathrm{t}^{\prime}\right)
\end{aligned}
$$

Here $H$ is the Hamiltonian. Since $H$ is a sum of the kinetic energy $\mathbf{p}^{2} / 2 m_{0}$ and the electromagnetic energy, it follows that

$$
\mathrm{H}(\mathbf{p}-\hbar \mathbf{k})=\frac{\hbar^{2} \mathrm{k}^{2}}{2 \mathrm{~m}_{0}}+\mathrm{H}(\mathbf{p})-\frac{\hbar \mathbf{p} \cdot \mathbf{k}}{\mathrm{m}_{0}} .
$$

Now, using the Campbell-Baker-Hausdorff formula [23] to first order in $\mathbf{p}$, we can show that 


$$
\exp \left(\mathbf{i k} \cdot \mathrm{y}_{2}\right) \exp \left(-\mathrm{ik} \cdot \mathbf{y}_{1}\right)=\exp \left(-\frac{\mathrm{i} \tau \hbar \mathbf{k}^{2}}{2 \mathrm{~m}_{0}}\right)\left[1+\mathrm{ik} \cdot\left(\mathbf{y}_{2}-\mathbf{y}_{1}\right)\right]
$$

Thus

$$
\left.\mathbf{Y}=\frac{1}{2}\left\{\exp \left(-\frac{\mathrm{i} \tau \hbar \mathbf{k}^{2}}{2 \mathrm{~m}_{0}}\right)\left(1+\mathrm{ik} \cdot\left(\mathbf{y}_{2}-\mathbf{y}_{1}\right)\right)\left(\dot{\mathbf{y}}_{1}-\frac{\mathrm{ck} \lambda}{2}\right)\right\}+[\mathbf{k} \rightarrow-\mathbf{k}]^{+}\right\}
$$

Noting that odd power in $k$ vanishes after $d^{3} k$-integration and $\left\langle k_{x}{ }^{2}\right\rangle=\frac{1}{3} k^{2}$, one obtains (keeping only terms linear in y)

$$
\mathbf{Y}(\mathrm{t}, \tau)=\dot{\mathbf{y}}(\mathrm{t}-\tau) \cos \left(\lambda \mathrm{c} \tau \mathrm{k}^{2} / 2\right)-\frac{\mathrm{k}^{2} \mathrm{c} \lambda}{6}(\mathbf{y}(\mathrm{t})-\mathbf{y}(\mathrm{t}-\tau)) \sin \left(\lambda c \tau \mathrm{k}^{2} / 2\right) .
$$

The linearized electron's equation of motion in nonrelativistic quantum mechanics is then given by inserting Eq. (37) into Eq. (4). Expanding the operator $y(t-\tau)$ in Eq. (37) in a Taylor series around $\tau=0$ and inserting it in Eq. (4), an equation involving a sum of derivatives of $y(t)$ is obtained. It can be shown that the coefficients of these derivatives are exactly those derived in Moniz and Sharp.

In particular, the coefficient of $\ddot{y}(t)$, the quantum mechanical self-mass $\delta \mathrm{m}$, is found to be

$$
\delta \mathrm{m}=\left(\frac{2}{3} \frac{\mathrm{e}^{2}}{\mathrm{c}^{2}}\right)\left(1+\frac{\lambda}{6} \frac{\partial}{\partial \lambda}\right)\left(1+\lambda \frac{\partial}{\partial \lambda}\right) \mathbf{\Omega}_{0}
$$

Here

$$
\Omega_{0}=\frac{2}{\pi} \mathrm{P} \int_{0}^{\infty} \frac{|\tilde{\mathrm{f}}(\mathrm{k})|^{2}}{\left(1-\lambda^{2} \mathrm{k}^{2} / 4\right)} \mathrm{dk},
$$

where $P$ denotes the principal value integration. Equation (39) is remarkable in that the self-mass remains finite in the point particle limit $\mathrm{f}(\mathbf{k}) \rightarrow 1$. In fact, the self-mass vanishes in this limit! $\delta \mathrm{m} \rightarrow 0$ ( $\mathrm{a} \rightarrow 0$ with $\lambda$ fixed in quantum mechanics).

Therefore, $\lambda$ (bare mass) $=\lambda$ (observed mass). This is why we used the same notation for these two Compton wavelengths.

In another limit, $\lambda \rightarrow 0$,

$$
\Omega_{0}=\frac{2}{\pi} \mathrm{P} \int_{0}^{\infty}|\tilde{\mathrm{f}}(\mathrm{k})|^{2} \mathrm{dk},
$$

and the theory reproduces the classical result, as it should. 
Whether the quantum mechanical equation of motion exhibits runaway or acausal behavior can again be studied by writing the quantum mechanical equation of motion in a Green's function form, similar to that at the end of section 4. Such an analysis in the point particle limit was carried out by Moniz and Sharp [9], who found that the motion is causal with no runaways if

$$
\alpha=\frac{\mathrm{e}^{2}}{\hbar \mathrm{c}}=\frac{\mathrm{r}_{\mathrm{e}}}{\lambda}<1.75 .
$$

In quantum mechanics, an electron is spread over a Compton wavelength. Thus, the above condition is reasonable in view of the classical causality condition Eq. (29), which can be written as $r_{d} / a<1.5$.

Nonrelativistic quantum mechanics is not valid for rapid motion with frequency $\hbar \omega \gtrsim \mathrm{mc}^{2}$ or if we approach a distance $\lambda$ near the electron, and a full QED analysis must be performed. The only calculation by QED was reported by Low [11]. He has not derived an equation of motion, but he has shown that the motion is finite in each order of $\alpha$. This is reasonable in view of the well-established renormalization theory that gives a finite answer for any physical process in perturbation expansion in $\alpha$. However, the perturbation theory is not suitable for taking the classical limit, and it is unclear whether QED can actually produce an equation of motion.

\section{CONCLUDING REMARKS}

The impression one gets from reading text books is that the classical electron theory is in trouble due to runaway solutions and acausal behavior. However, we have seen that classical theory is actually fine if the electron is taken to be a spherical object of not too small a radius, greater than the classical electron radius $r_{e}$. The restriction is reasonable since $r_{e}$ is about 100 times smaller than the Compton wavelength $\lambda$, and we cannot consider distances less than $\lambda$ without considering quantum mechanics.

The nonrelativistic quantum theory also looks fine: In fact, the quantum theory is better behaved than the classical theory because it is finite and causal irrespective of the size of the electron, as long as $\alpha=r_{e} / \lambda<1.75$. The inequality is certainly satisfied in the real world where $\alpha=1 / 137$. The fact that the quantum theory is better behaved is also reasonable because the electron is smeared out due to the uncertainty principle. The quantum theory as reviewed here has the appropriate feature of having the correct classical limit. However, the limit of validity of the nonrelativistic quantum analysis is not really understood. The vanishing of the self-mass $\delta \mathrm{m}$ in the point-particle limit must be accordingly interpreted with care.

In fact, we know that the nonrelativistic treatment cannot strictly be valid in the point-particle limit because of vacuum polarization. It is reasonable that a full QED calculation will be at least as well-behaved as is the case in nonrelativistic quantum 
mechanics. Unfortunately, there exists as yet no real calculation in QED to confirm these conjectures.

\section{ACKNOWLEDGMENTS}

I would like to thank Andy Sessler for collaboration on reference [1], on which this paper is based. I would also like to thank Dave Jackson, Francis Low, Robert Mills, Fritz Rohrlich, and David Sharp for useful remarks.

\section{REFERENCES}

1. Kim, K.-J., and Sessler, A.M., "The Equation of Motion of an Electron," ANL preprint LS-270 (1998), Proc. $8^{\text {th }}$ Workshop on Advanced Accelerator Concepts," July 5-11, 1998, Baltimore, MD.

2. Abraham, M., Theorie der Elektrizitat, Vol II: Elektromagnetische Theorie der Strahlung, Leipzig, Teubner (1905).

3. Lorentz, H.A., The Theory of Electrons, Leipzig: Teubner (1909) (2nd edition, 1916).

4. Poincaré, H., " On the dynamics of the electron," Rendiconti del Circolo Matematico di Palermo 21, 129 (1906); translated by Scientific Translation Service, Ann Arbor, MI. The article is also translated into English with comments in "On the articles by Henri Poincaré," A.A. Logunov (Publishing Dept. of the Joint Institute of Nuclear Research, Dubna, 1995).

5. Dirac, P.A.M. "Classical theory of radiating electrons," Proc. Roy. Soc. Lond. A167, 148 (1938).

6. Sommerfeld, A., "Simplified deduction of the field and the forces of an electron moving in any given way," Akad. van Wetensch. te Amsterdam 13 (1904) (English translation 7, 346 (1905)).

7. Page, L., Phys. Rev. 11, 376 (1918).

8. Caldirola, P., “ A new model of classical electron," Nuovo Cimento 3, Supplemento 2, 297 (1956).

9. Moniz, E.J. and Sharp, D.H., " Radiation reaction in nonrelativistic quantum electro-dynamics," Phys. Review D 15, 2850 (1977); see also Grotch, H., Kazes, E., Rohrlich, F. and Sharp, D.H., "Internal retardation," Acta Physica Austriaca 54, 31 (1982).

10. Kim, K.-J., to be published.

11. Low, F., "Run-Away Electrons in Relativistic Spin 1/2 Quantum Electrodynamics," Preprint MITCTP-2522 (1997).

12. Panofsky, W.K.H., and Phillips, M., Classical Electricity and Magnetism, Reading, MA: AddisonWesley, 1962.

13. Jackson, J.D., Classical Electrodynamics, New York: Wiley, $2^{\text {nd }}$ Edition, 1975; $3^{\text {rd }}$ Edition, 1998. Chapter 16 of $3^{\text {rd }}$ Edition contains a review of the equation of motion of an electron, much more extensive than in previous editions.

14. Rohrlich, F., Classical Charged Particles, Reading, MA: Addison-Wesley (1965) (2nd edition 1990).

15. Yaghijian, A.D., "Relativistic Dynamics of a Charged Sphere," Lecture Notes in Physics 11, Springer-Verlag, Berlin (1992)

16. Pearle, P., "Classical Electron Models," in Electromagnetism: Path to Research, D. Teplitz, Ed., New York: Plenum, 1982.

17. Rohrlich, F. “ The Dynamics of a Charged Sphere and the Electron," Am. J. Phys. 65, 105 (1997).

18. Schott, G.A., Electromagnetic Radiation, Cambridge University Press (1912).

19. Schwinger, J., "Electromagnetic Mass Revisited," Foundations of Physics, 13, 373 (1983).

20. Boyer, T.H., Phys. Rev. D25, 3246 (11982).

21. Baier, V.N., and Katkov, V.M., Sov. Phys. JETP 26, 854 (1968).

22. Cahn, R.N., and Jackson, J.D., unpublished Lawrence Berkeley National Laboratory note.

23. Dragt, A.J., and Finn, J.M., J. Math. Phys. 17, 2215 (1976). 